\title{
MARITIME WORK AND COMMUNICATION
}

\author{
Peter Bogh Andersen, \\ Department of Computer Science, \\ University of Aalborg

\section{INTRODUCTION}

This paper presents findings from the project "Elastic Systems" under the Danish Center for Human Machine Interaction. The project aims at developing methods for analyzing dynamic maritime work processes and for designing flexible instrument interfaces that will support changing work environments.

Here I only address the former issue, the design issues being published elsewhere, e.g. in Andersen(1999) and Andersen \& May (2001). More data can be found in Andersen(2000).

The purpose of the present paper is twofold: (1) to present characteristics of maritime work and communication, and (2) to suggest a conceptual framework that covers communicative as well as non-communicative acts.

The latter purpose is motivated in two ways. Theoretical motivation: we know that language is self-referential, so that it can speak of non-linguistic entities like ships and waves as well as its own properties, such as the correct wording or style. The easiest way to accomplish this is for language to treat both domains in a similar way. The other motivation is more practical: it is a fact that communication and physical actions are interwoven in maritime work, and a theory that builds on a sharp distinction between these two kinds of behaviors will miss this basic characteristic.

The data is from a voyage aboard Sally Maersk from Algeciras to Göteborg and back to Rotterdam. We recorded 60 hours of high quality video, and the paper builds upon a 16 hours trip from Felixstowe to Rotterdam, supplemented by data from simulated voyages in the simulator at the Danish Maritime Institute.

The conceptual framework is based on Lind 1994 and Lind 2000 and distinguishes between the following types of action types:

(1) Prevent(suppress, avoid): if I hadn't done it, then an undesirable state would have developed. Prevent drifting by using auxiliary rudder.

(2) Maintain: if I hadn't done it, then a desirable state would have disappeared. Maintain course.

(3) Help: a positive state would have developed anyway, but $\mathrm{I} / \mathrm{something} \mathrm{made} \mathrm{it} \mathrm{happen} \mathrm{faster/more}$ efficiently. The wind helped us moving the ship to quay.

(4) Create(produce): if I hadn't done it, it wouldn't have happened. Turn on the thrusters.

(5) Destroy(remove): if I hadn't done it, a undesired state would have persisted. Stop engine.

(6) Let: it will happen even if I don't do anything. Let the ship slide into berth without using power.

The meaning concept implied here is a modal one, since all action types are defined by means of counterfactual statements: what would happen, had we not done anything, a course of events termed "passive projection" in Ryan 1991. This modal conception of meaning is among the stock of trade in structuralist linguistics: meaning units are constituted by means of differences and are defined negatively against units that are possible now, but not actualized. Saying that something is blue is to negate that it is green, brown, yellow, etc.

The German sociologist Niklas Luhmann has a meaning definition very much in agreement with this. To Luhmann, meaning is a specific mode of experience: namely that human experience, although always filled with a specific actual content, is still aware of the possible contents that are left out:

Experience experiences itself as variable [...]. It does not find itself closed and self-contained, not restricted to itself, but is always referring to something that is at the moment not its actual content. [...] The problem of integrating the actuality of experience with the transcendence of its other possibilities remains inescapable, and inescapable, too, is the form of experience processing that accomplishes this. It is this that I call meaning.

Luhmann 1990: 25

A linguist would say that meaning is structured in paradigms only one member of which can be chosen, while the rest of the paradigm is present for the consciousness and affects its actual contents.

Meaning is also characterized by its immense complexity that cannot be handled by the individual system:

The most important feature of the differentiation between Actuality and Potentiality found in experience resides in the character of the overabundance of possibilities, which by far exceeds what can be realized through action or actualized in experience.

Luhmann 1990: 26

Therefore, actualizing a specific meaning presupposes negating the rest.

The constitution of this world of not-yet-actualized potentialities, which is constantly given and accompanies all experience, rests on the specifically human capacity for negation......] Negation appears [...] to actually constitute the universality, i.e. the world reference, of all practical life - especially where experience or action is directed 
positively toward some particular meaning and intends it under the form of "is" or "ought". Luhmann 1990: $27-$ 28

\section{AN OVERVIEW OF MARITIME WORK AND COMMUNICATION}

In this section, we give a broad overview of characteristics of maritime work and communication, as it can be observed on the bridge. The next sections give examples and suggest ways of analyzing the findings by means of the framework in Section 1.

\section{Work:}

- Purpose. The purpose of bridge work is to move the ship from departure to destination (produce) in a safe (prevent) and efficient way and with cargo intact (maintain). From this formulation particularly three concepts seem relevant: Goal (arriving at destination), Maintenance (maintaining cargo and ship), and Avoidance (avoiding accidents).

- Tasks. Bridge work fall into two intertwined tasks: Maneuvering is the art of pitting the controllable forces (such as rudder and propeller) against the uncontrollable ones (such as wind and current) in order to achieve one's purpose (DMI 1995: 1). Navigation is the art of fixing the correct position of the ship.

- Division of labor. There is a strict division of labor defined by the hierarchy $\left(2^{\text {nd }}, 1^{\text {st }}\right.$ and chief officer, captain) and the watch schedule. However, planning and execution are not disjoint since the captain who is responsible for the planning is also in charge in critical situations.

- Reciprocity. The officer must be able to reconstruct the intentions of the captain on the foreign ship, and imagine how the foreign ship conceives of himself.

\section{Control}

- Control chains. Bridge work involves chains of control that change regularly during the voyage and which involve human and mechanical components. Control changes can be simple or complex. Controlactions can be the target of other control actions, such as maintaining telephone lines in order to prevent accidents.

\section{Communication}

- Uniformity: Conversations are heterogeneous with respect to register, time and medium. Greetings of the pilot could occur in any register whereas the highly specific rudder- and course commands belong to the maritime domain. Some conversations take only a few seconds, others last several hours. Some conversations are oral, others go through walkie-talkie or intercom, whereas still others take place over VHF radio.

- Standardization. Conversations are to some degree event-driven. The structure of some utterances is not primarily controlled by language-internal rules, but by events outside the control of the crew, or by the physical working process itself. Conversations range from fixed protocols to more free interchanges.

- Time-structure. Conversations are intertwined and concurrent. The crew often maintains several threads of conversations at the same time and when the current conversation is interrupted, another can be resumed.

- Verbalization. Work, including communication itself, is verbalized. You must say what you are doing. It is important that the crew maintains an updated overview of the state of affairs (Carstensen \& Nielsen 1998), and for this reason, crew members must verbalize their actions. It is also important that errors do not go unnoticed, and it is particularly important that authority does not suppress criticism from subordinates of wrong decisions.

- Meta-communication. Meta- and object-communication - communicating about communication and communicating about the voyage - are mixed. There are chains of communication where a speaker request another speak to ask a third speaker to....

- Reduction of complexity. Complexity is reduced by focusing only on a few choices in the individual conversation. Complexity is also reduced by relying on fixed protocols.

\section{WORK}

As mentioned in Section 2, three kinds of actions are important in maritime work: bringing the ship to its destination, maintaining the cargo intact and avoiding casualties. 


\section{Purpose and task structure}

If we look closer at the six action types in Section 1, we discover that they imply different set-ups of auxiliary forces. Produce and destroy are actions that change the world into a state that would not obtain, had we not intervened. They therefore imply that certain means are employed to effect this change.

Maintain and avoid make the opposite assumption, namely that there are forces in the world that will change it, if we do not do something. Furthermore, the means we employ in these action types must be able to counteract the forces. In Figures 3.4 - 3.9 below we use the three types of relations shown in Figures 3.1-3.3 as building blocks. (In many cases, the relations in Fig. 3.2 and 3.3 can be analyzed as control actions in their own right. Solid line: the actor's intention. Dashed line: the course of events, had the actor not acted.)

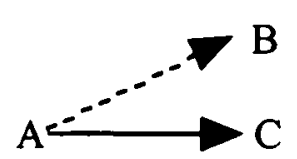

Fig. 3.1. A changes into $B$ unless an action is performed which will result in the outcome $C$.

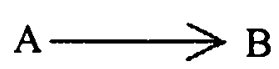

Fig. 3.2. A furthers the outcome of state $B$ or the proper working of object B

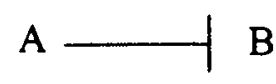

Fig. 3.3. A counteracts the outcome $B$ or the proper working of object

B

The six action types can then be diagrammed as shown in Figures $3.4-3.9$. For example, to produce is to see to it that a state that would otherwise be absent in fact becomes present. And to destroy is to cause something otherwise present to become absent.

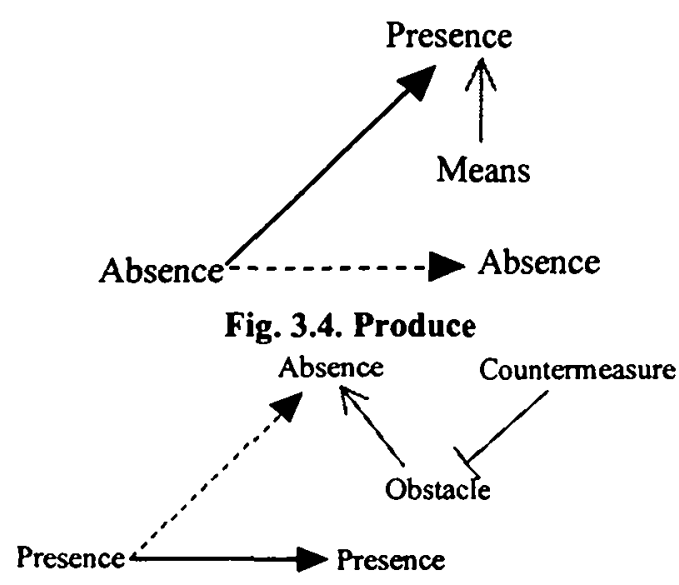

Fig. 3.6. Maintain

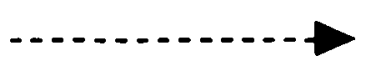

Fig. 3.8. Let happen.

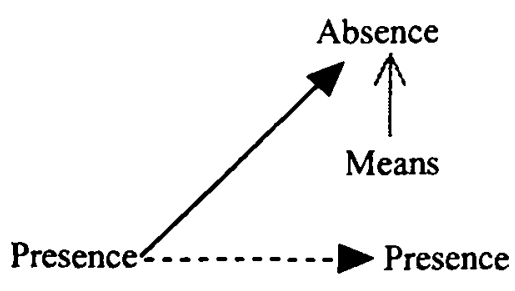

Fig. 3.5. Destroy

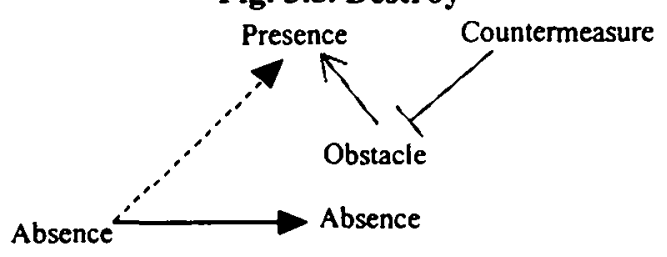

Fig. 3.7. Suppress

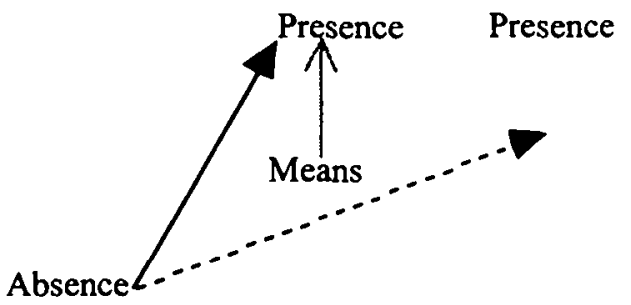

Fig. 3.9. Help happen.

Countermeasures can work in two ways. Either the force of the obstacle is weakened as shown in Figs. 3.6 and 3.7: an example is the building of breakwaters in harbors to counteract the force of the waves. Or the countermeasure influences the same state variable as the obstacle, but in the opposite direction. An example is countering side-wind by means of permanent rudder. In this case both obstacle and countermeasure influences the same state-variable, namely the course of the ship.

As an example of a authentic configuration of forces, consider the maneuver discussed in Text 3.1. 


\begin{tabular}{|l|l|}
\hline (C) & $\begin{array}{l}\text { Look, now you must also take care, look, now, we reduce speed, right, then the wind gets } \\
\text { more power over the ship and ... }\end{array}$ \\
\hline (A) & Yes \\
\hline (C) & $\begin{array}{l}\text {...and starts to, so...there is possibly current too, so just give it about five degrees drift to } \\
\text { port. }\end{array}$ \\
\hline (A) & We are already high up, we steer seventy, so ... \\
\hline (C) & Yes \\
\hline (A) & $\begin{array}{l}\text { And the course in the map says seventy eight....but it did take hold when I came over } \\
\text { Noordhinder }\end{array}$ \\
\hline (C) & $\begin{array}{l}\text { Yes, but it changes all the time here, right, but particularly, particularly when you reduce, } \\
\text { reduce speed, then you of course get more sensitive to ... }\end{array}$ \\
\hline (A) & Yes \\
\hline (C) & Both wind and current \\
\hline (A) & That is evident \\
\hline
\end{tabular}

Text 3.1. Complex control.

When speed is reduced, the rudder is less effective since the force of the rudder is dependent on the water flowing past. At the same time, the movement of the ship is influenced by currents which necessitates auxiliary rudder. So when speed is reduced, more auxiliary rudder must be given in order to stay on course.

Or, using our building blocks (Fig. 3.10): we want to maintain the course, but the wind will change it if we do not intervene. We can use the rudder as a means for counteracting the force of the wind, but, at this time, we are slowing down to kill time, so the low speed will decrease the force of the rudder. In such cases, we can use the engine to speed up a bit to counteract the effects of low speed (a more detailed analysis will show that what really happens is that faster rotation of the propeller increases the flow of water along the rudder and thereby increases its effect).

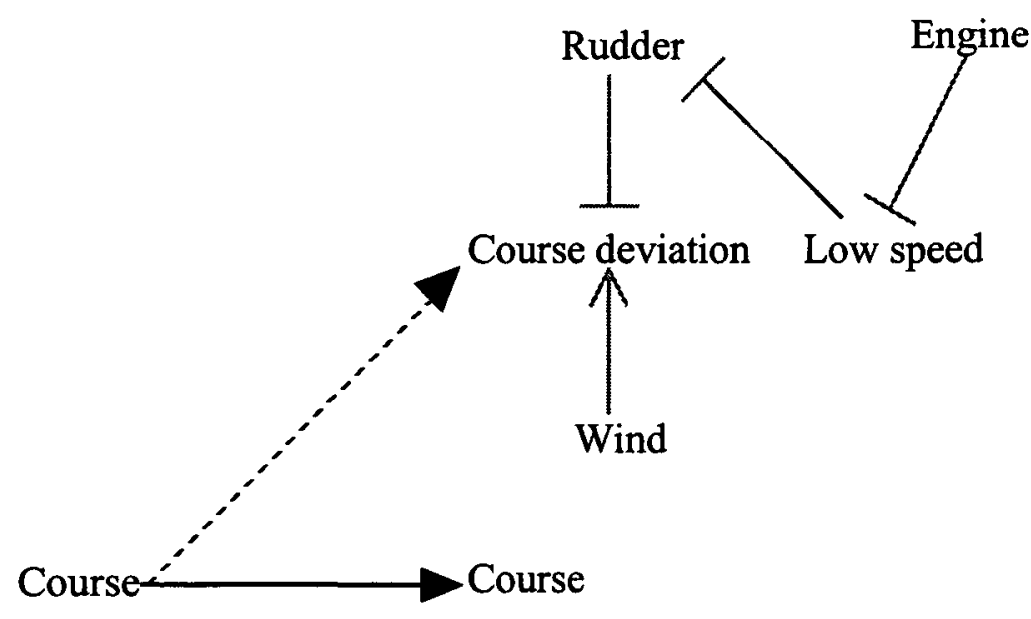

Fig. 3.10. Maintaining course. Speeding up to increase the effect of the rudder in order to counteract the force of the wind.

Many maritime maneuvers can be represented by the configuration shown in Fig. 3.11. The means aims a producing the goal state and may simultaneously produce the negative side-effect. The side-effect and the obstacle suppress the achievement of the goal, and the countermeasures suppress the effect of obstacle and sideeffect. 


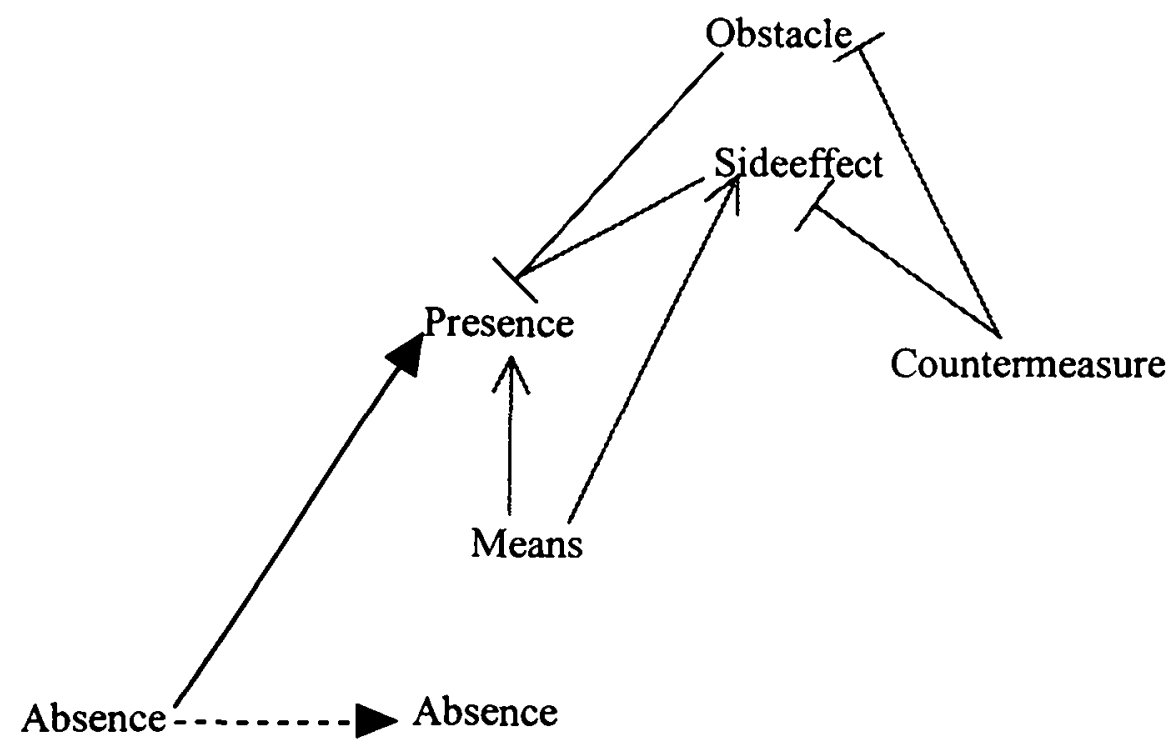

Fig. 3.11. Goal, means, side-effects, obstacles and countermeasure as a set of connected control actions.

Fig. 3.12 shows an example of Fig. 3.11: The rudder is used to counteract a turning moment produced during a crash stop that is achieved by putting the propeller in reverse.

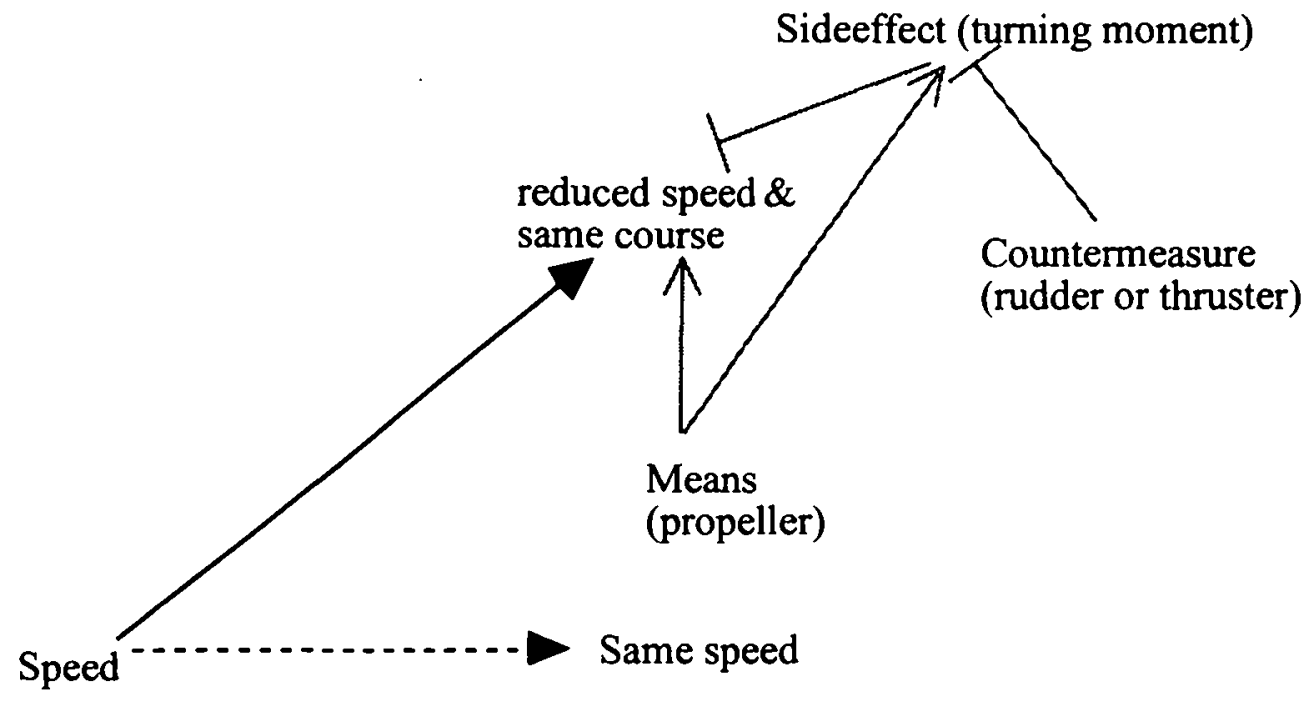

Fig. 3.12. Using rudder to counteract turning moment produced during crash stop effected by putting the propeller in reverse.

Real maneuvering and navigation consist in handling a large set of concurrent control actions where the same objects and states participate in several control actions simultaneously.

The countermeasures in Fig. 3.11 does no only show up in maneuvers, but also in the layout of the bridge and in communication protocols. Therefore, merely knowing the purpose of an instrument does not suffice, one also has to know the type of accidents it is designed to prevent. For example, both bridge and engine can control the engine power. This shared control is regulated in order to prevent failures. In a cargo ferry, engine control could be exercised from five different places: the main distinction was the opposition between bridge and engine room; if control were on the bridge, it could be allocated to four different places: either to the stern bridge or, on the front bridge, to the central console or to the port or starboard consoles. See Fig. 3.13.

The bridge crew could itself physically distribute control to the four "bridge-locations but not to the engineer. In the latter case, the bridge can only request that the engine takes over control. The engineer acknowledges the request and itself makes the physical transfer of control in the engine room (Fig. 3.14).

Why this protocol? The reason is that the ship's safety is jeopardized if the engineer happens not to be present in the control room of the engine at the moment control is transferred. In this case, nobody would be in control of the ship. 


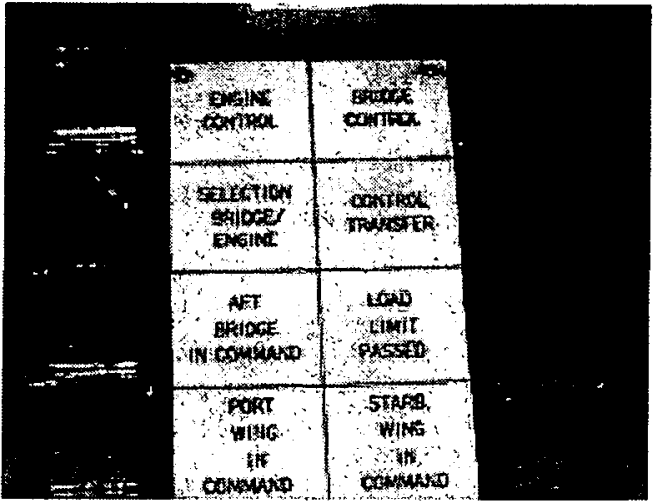

Fig. 3.13. Control transfer panel on bridge.

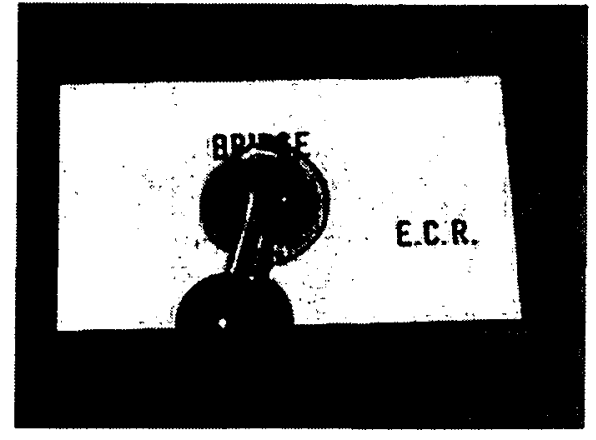

Fig. 3.14. Control switch in engine room

Some of the communicative patterns conform to the pattern in Fig. 3.11 as well. For example, commands are normally repeated (Text 3.2), and this is true also of reports via VHF or intercom (Text 3.3). The purpose is to avoid misunderstandings due to noise.

(L) And the bow to port please ()

(C) Bow full to port

(L) Yes ... dead slow astern

(C) Dead slow astern

Text 3.2. Repeated orders during berthing in Rotterdam. $L=$ pilot, $C=$ captain.

\begin{tabular}{|l|l|}
\hline (A) & and then the stern is inside the comer of the quay (radio) \\
\hline (C) & you've got the stern inside, OK (radio) \\
\hline (C) & the stern is inside the corner \\
\hline (L) & Yeah, okay, okay \\
\hline
\end{tabular}

Text 3.3. Repeated reports during berthing in Rotterdam. $L=$ pilot, $C=$ captain, $A=2^{\text {nd }}$ officer on the stern deck.

Fig. 3.15 shows how the task structure is repeated in the communication protocols. Noise may hinder the utterance in producing the desired action, and repetition counteract this negative effect.

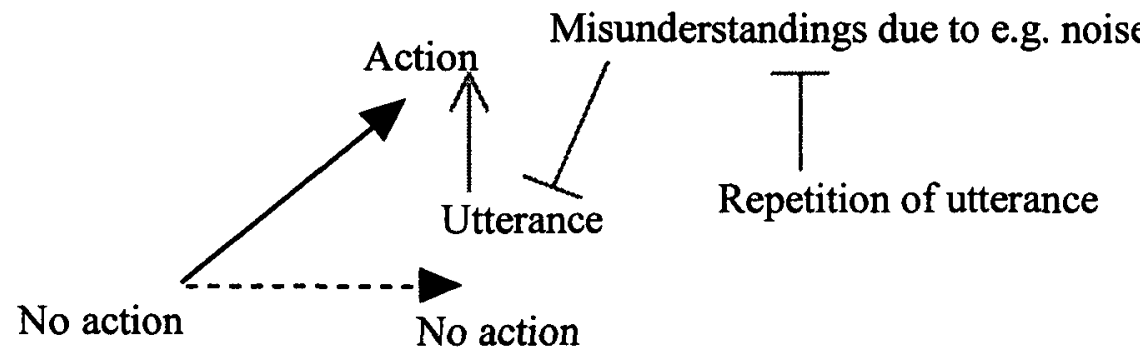

Fig. 3.15. Goal, means, side-effects, obstacles in communication.

The importance of obstacles and negative side-effects is due to the fact that many forces working on the ship are uncontrollable. For the experienced officer, some configurations of these forces are predictable while others are not, some being chaotic (in the mathematical sense).

Text 3.1 gave an example of forces that can be partially predicted, but inside harbor the forces may turn out to be unpredictable:

(B) It is possible it was that which, or...the pilot talked about it being deeper the last distance to the quay, (...) than it really is out in the channel (...) he said that at arrival (...) he kept saying that strange things happen which you do not exactly know, (...) before you are in it, so there is only one way, and that is to keep your eyes open, and then...

Text 3.4. Unpredictable forces. 


\section{Planning and execution}

Apart from being responsible for the planning and the decisions, the captain also takes part of the execution since he is in control during departure from and entrance to harbors (the helmsman is taking care of the wheel, the captain wields the machine telegraph, and the pilot guides both). This is unique for the maritime domain, since in most organizations planning and execution are done by different persons. This means that the officers participate in two rather different kinds of conversations about the same event, planning and execution.

Let us first look at the planning bit.

(C) And then .. I, I guess we go in the normal way and..

(C) And turn around in the Beer Canal ..

(L) That's right

(C) And and back her in

(L) Back her in yes yes

(C) Yeah yes

$\ldots$

(L) (..) procedure here to back (...)

(C) That's more or less normal procedure now (...)

(L) That is normal procedure for this type (...)

(L) Yeah yes yes

(C) Yeah yes yes

(L) We we could turn here but yeah

(C) I have tried it with the K-class, to turn it here, get the bow up, and well, it was nice and calm so ... no problem

(L) That is right for the wind is pushing your all the way back towards the buoy here so ...

(C) Yeah ... yeah

(L) Now I prefer to do it with this these type always here in time (...)

(C) Yeah you don't get anything

(L) No you don't get anything

(C) No no

(C) So what do you know, what kind of wind we have in there, we have about what here, eight meters per seconds out here now

(L) Yeah it has been force five from Northeast something like that, I think so yes

(C) Can we guess, um can we berth her without a tug?

(L) Arh I think one aft

(C) One aft

(L) Yes

(C) Yeah

Text 3.5. Planning the entrance to Rotterdam at 10.50 .

At the planning stage at 11 o'clock, the maneuver is characterized by the opposition, turn and back, and is presented as the normal and safest way, "you don't get anything". The reference to "normality" is probably not a coincidence, but may be a way of reducing meaning complexity in Luhmann's sense (Section 1). Both pilot and captain have tried this before many times, and by referring to a "standard", many decisions have been taken without needing discussion.

The text has expressions for probability interspersed since neither captain nor pilot want to impose on the other:

I guess, we could, I prefer, Can we berth her without a tug? Arh I think one aft.

and is in the future or the past (I have tried it with $\mathrm{K}$ - class). They cooperate about the planning, supporting and supplementing the utterances of the other, and taking care to be polite and supportive. The captain may have wished to leave out the tug to save money, but does not insist when the pilot recommends one.

The execution of this plan looks quite different four hours later. 
(L) And the stern thruster to starboard Captain

(C) Stern thruster to starboard

$$
\text { (P) }
$$

(L) Stop engine

(C) Stop engine

$$
\text { (P) }
$$

(C) Four knots

(L) Yeah that is okay

(C) Yeah ... that's okay

(L) Is she always working?

(C) The thruster?

(L) The thruster yeah

(C) Yeah

(L) To starboard, ah the stern thruster to starboard and the bow to port

(C) Bow to port yeah

$$
\text { (P) }
$$

(L) Mmm she is not swinging that much

(C) No only

(L) Ah few degrees only

(C) Few degrees only

(L) All right ... hard .. hard to port please

(C) Hard-a-port

(L) Dead slow ahead

(C) Dead slow ahead

$$
\text { (P) }
$$

(A) And then the stern is inside the comer of the quay (radio)

(C) You've got the stern inside, OK (radio)

(C) The stern is inside the corner

(L) Yeah, okay okay

(L) See the wind have a lot of effect on the ship arh?

(C) Yes ... it is also (...) up now fifteen $\mathrm{m} / \mathrm{sec}$, that is thirty knots

(L) Yeah

(P)

(C) And it is nearly on the beam still

(L) Yeah

(P)

(L) Okay stop the engine

(C) Stop engine

(L) Midships the wheel

(C) Midships
(P)

(L) And stop the stern thruster captain

(C) Stop the stem thruster

(P)

(C) Stern thruster stopped

(L) Okay

(L) $\quad$ Stop the bow thruster

(C) Stop the bow

(P)

Text 3.6. Execution (turning the ship) at 14.11

The two tasks, turn and back, are dissolved into many small steps characterized by rudder, thruster, and machine telegraph orders (midships the wheel, stern thruster to starboard, dead slow ahead), and occasional reports of 
the state of the ship (Is she always working? She is not swinging that much). Probability has turned in necessity, and arguments have vanished. Verbs are normally omitted (except in stop engines) and only sentence parts designating instrument settings (dead slow astern) remain. The tense has become present. The utterances follow a rigid Order + Accept schema, and are intermingled with physical manipulation of controls and observation of instruments and environment.

\section{Reciprocity}

During departure from Felixstowe (Text 3.7), captain and pilot identify a vessel and begin guessing at his course (what course he is gonna take, he is most on, on the western side of the fairways). Predictability seems to be highly valued, since they complain about ships that cut straight over, and the captain enquires whether she will have a pilot aboard, implying that a pilot will ensure normal and predictable behaviour.

(L) Okay ... ferry coming in there

(C) Yeah he is right here

(L) Small ship there

(C) Yeah

(L) And the dredger

(C) No that is the dredge..

(L) That is the dredger, and there is a small ship (...) coming in or .. I'm not quite sure what course he is gonna take, but we will se in a minute

(P)

(C) Yeah its looks like, he is most on on the western side of the fairways

(L) Yeah

(C) Yeah
(P)

(C) But some (...) when when the draft allow us into the (...)

(L) (...) cuts straight over and all sorts of thinks yeah

(C) Yes I have seen that happened

(L) Yeah

(P)

(C) Is he coming in without a pilot....

(L) He is coming without a pilot yeah, he is gonna pick up a Pilot ... in here, to go to Ipswich, which is sometimes a bit of a problem cause they don't always ...

(C) Yeah I can imagine

(L) Realise exactly where they should be in the channel

(C) Mmm mmm

(P)

(L) Nah, well as long as he keeps heading like that, it will be all right

Text 3.7. Guessing the intention of a small ship. Departure from Felixstowe.

The reason is that the captain and pilot keep imagining scenarios for their own near future, and in order to do that, they have to make guesses about the intentions of other ships. Most of the times they succeed, but sometimes the guesses are wrong.

In Text 3.8, the foreign captain believes that Sally will pass her head-on, whereas we are in fact turning, so that the action will be "overtaking". The pilot does not realize the error, but the captain does. Originally they believed that the foreign captain believed that they intended to pass by "overtaking", but the captain realizes that the foreign captain believed them to intend to pass head-on.

$\begin{array}{ll}\text { S.Atlantic } & \text { Standing by zero two one six[VHF] } \\ \text { Pilot } & \text { Okay [VHF] } \\ \text { Master } & \text { Who was that? } \\ \text { Pilot } & \text { The outgoing ship, Sealand Atlantic, she want to pass port to port } \\ \text { Master } & \text { How, how can we do that? [pass the Sealand Atlantic port to port] } \\ \text { C. officer } & (\ldots) \\ \text { 1. officer } & \text { Yes } \\ \text { Pilot } & \text { We are steering around slowly, slowly } \\ \text { Pause } & \\ \text { 1. officer } & \text { I presume he means that he will ... }\end{array}$




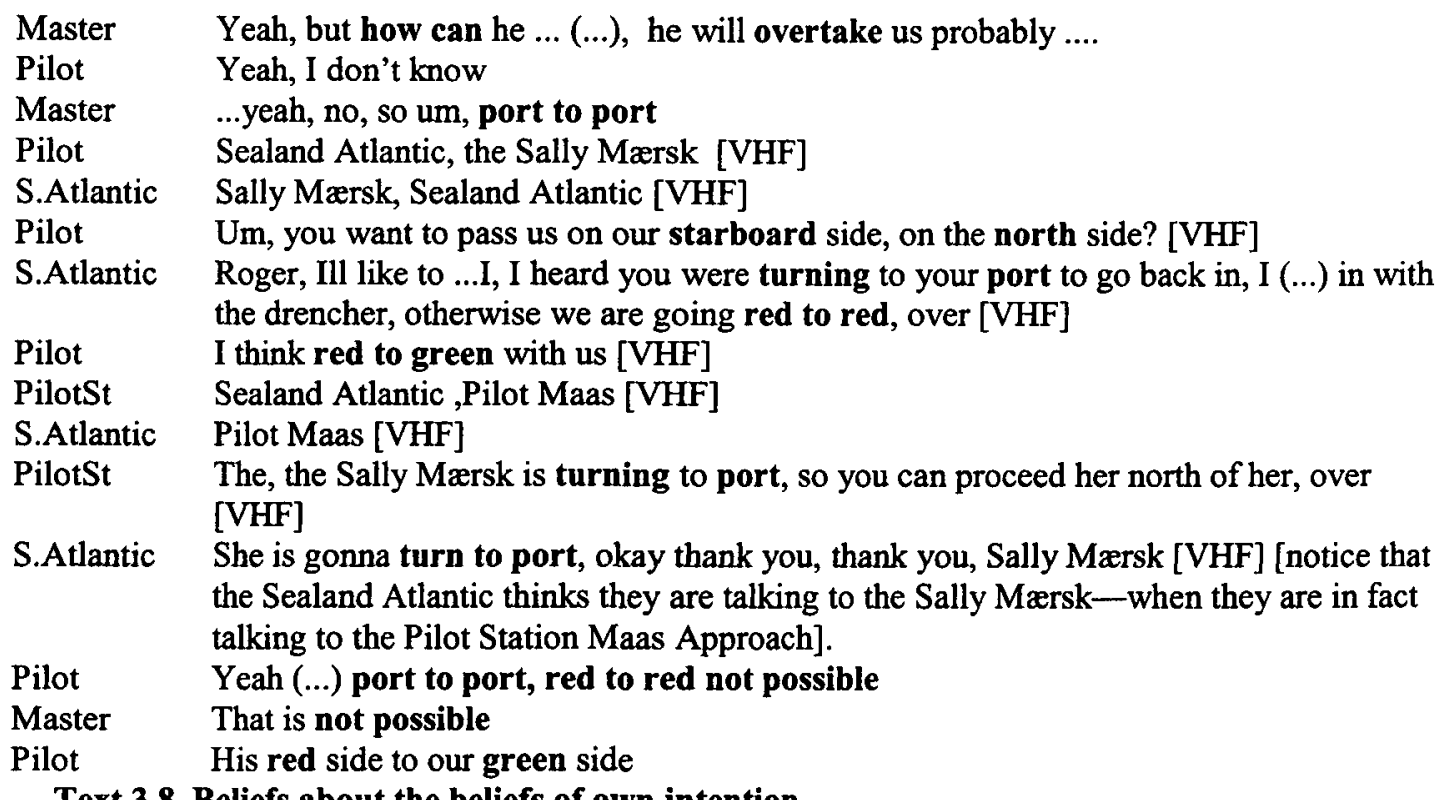

Text 3.8. Beliefs about the beliefs of own intention.

The accident was prevented by the captain's being able to recursively having beliefs about the other's belief about his own intentions. The suggestion that meaning is recursive in this way can also be found in Posner (1993) and Ryan (1991).

\section{CONTROL}

It is characteristic of maritime work that it contains long control chains consisting of intermingled humans and non-humans that work in real time. Two simple control elements form a chain if the controllee of one equals the controller of the other.

The reason for these chains is that location is often a hindrance for performing a certain job on a ship. For example, during berthing, the captain must be on the bridge where the helm and machine telegraph are located, but from the bridge he cannot see the ship's sides, and he cannot manipulate the wires. In the old days, the problem was solved by cooperation and communication, so we had pure social chains of control. During the past decades, crew size has been halved, and remote control has replaced cooperation in many places, yielding mixed chains of control. The machine telegraph is an example: originally it was a device used by the captain to signal commands to the chief engineer. Nowadays it allows the captain to control the machine directly.

We characterize a chain by 5 elements:

1. Controller: the item from where control originate. Human or piece of machinery.

2. Mediator: the item through which control is administered. Language or piece of machinery.

3. Controllee: the item controlled. Human or piece of machinery.

4. Behavior: The controlled aspect of the Controllee. Verbal or non-verbal behavior.

5. Effect (optional): the ultimate causal effect of controlling the behavior.

An example of a complex heterogeneous chain is shown in Fig. 4.1. The pilot controls the helmsman's hands by means of language, and the helmsman controls the rudder engine by means of the wheel. The ultimate effect is to control the ship's course.

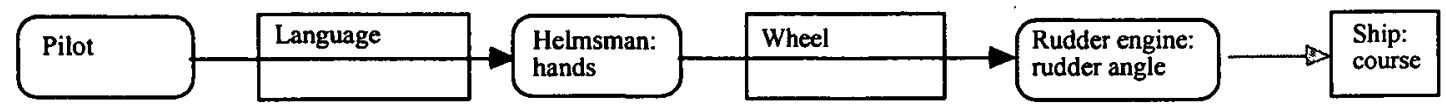

Fig. 4.1. Complex chain: pilot guiding helmsman. 
Text 4.1. exemplifies the language part of the chain.

(L) One zero zero please

(K) One zero zero

(L) Yeah

(P)

(K) One zero zero

(L) Thank you

Text 4.1. Complex, heterogeneous chain: pilot guiding helmsman

Another characteristic is that the control chains change regularly during entry and exit of harbor. In open water, there is only one person on the bridge who handles everything, whereas during entry, the complexity of cooperative arrangements increases: the pilot embarks and guides the captain, a helmsman is called to handle the rudder, the chief officer is called in, and finally control is moved into the wings where the captain finishes the approach.

Fig. 4.2 shows that the control chain changes from simple to complex when the helmsman is called in.

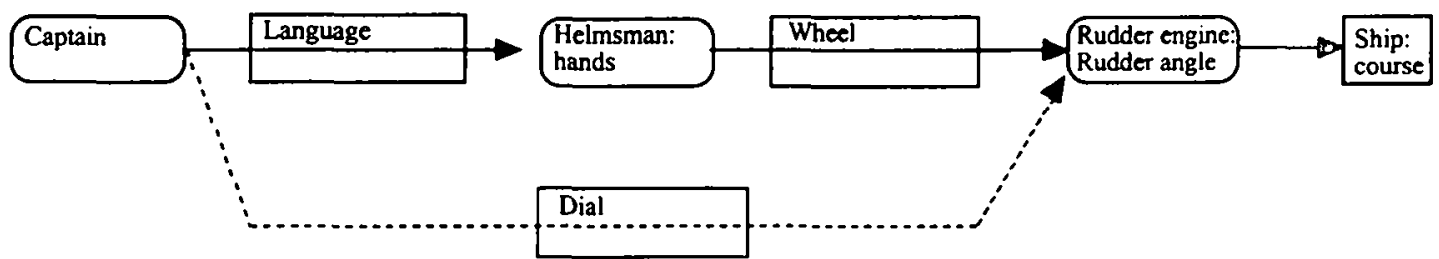

Fig. 4.2. Assigning and dismissing helmsman

Text 4.2 and 4.3 exemplify assignment and dismissal of the helmsman:

(P)

(C) Yes $\mathrm{O}$, will you take the wheel, now we will soon have some action

(O) (...)

(C) Zero nine five

(O) Zero nine five

(C) And it is yours

(L) Okay

(C) Yeah hand steering

(L) Yeah

(C) You have got hold of it, right, it works and everything.

(O) Yes

(C) Good

Text 4.2. Captain assigns Helmsman to duty.

(C) Yes, $\mathrm{K}$, I will let you off the hook, I have got it here now

(K) (...)

Text 4.3. Captain dismisses Helmsman.

The different types of control change are verbalized differently: change of medium (e.g. between wing and central bridge) is seen as a movement of control (Captain: Yes, you can take the steering in, and steady it at a hundred and thirty seven, one three seven. Officer: I have taken thruster and telegraph in) which is not the case when humans are involved. Here the social control structure influences the way the change is treated: captain pilot exchanges are like a negotiation where the parties must agree, whereas captain - helmsman exchanges are more like ordering as in Text 4.2; and in a pilot $-I^{\text {st }}$ officer exchange, the pilot suggests hand steering to the officer, who subsequently asks the captain if this is $\mathrm{OK}$.

In all cases, the change must be marked verbally in a clear and public way.

\section{COMMUNICATION}

Conversations are heterogeneous with respect to register, time and medium.

\section{Heterogeneity}


The highly schematized captain-helmsman commands in Text 4.1 are unique to the tasks of the maritime domain, whereas the polite greeting of the pilot in Text 5.1 could be heard in any social setting where a newcomer enters.

\section{(L) Good morning Sir}

(C) Good morning Sir

(L) How are you

(C) C is my name, welcome onboard

(L) Thank you

.... $\quad$....

(L) You allow smoking or not captain

(C) You are very welcome to smoke

(L) Thank you Sir

(C) I do not hate you for that reason Sir

\section{Text 5.1. Greetings.}

The short rudder commands (Text 4.1) take only a few seconds, whereas a planning and re-planning discussion, due to the fact that the ship occupying our berth was delayed, took 11 hours (the start is shown in Text 3.5).

\section{Triggers}

Some types of communication are triggered by internal linguistic rules. This is true of oral genres such as presentations and speeches, and of most written genres. There is nothing to interrupt the speaker/writer who can undisturbed compose his speech or text.

The situation is different in maritime communication: although linguistic rules do regulate local parts of the conversation, so that answers follow questions, and acknowledgements follow orders, the main structure of the conversation is triggered by outside events and the progress of work. This means that the coherence of communication resides outside language.

Longer stretches of communication are triggered by the environment and not by linguistic rules. This is obvious with the many comments on ships appearing and disappearing: such comments can only be made after the vessel is in view, and are uninteresting when it has sailed past.

(L) Okay ... ferry coming in there

(C) Yeah he is right here

(L) Small ship there

(C) Yeah

Text 5.2. Comment on passing ferry.

(D) I have him out in four [degrees?] to the starboard

(C) Mmm

(D) There is one coming towards us

(C) Yes

(D) CPA at zero comma four

Text 5.3. Comment on approaching ship.

(C) We can se her coming in the canal now or...

(L) Yes .. big container ship coming out with ... if it's Gudrun I don't know .. we will see

Text 5.4. Comment on approaching ship.

Thus, maritime communication is event-driven, i.e. it depends upon events that are outside the control of the crew. This means that there is no guarantee that one conversation can end before another one begins. In fact, the typical situation is that a conversation is interrupted by another one, and later resumed. In Text 5.5, the captain is involved in a long conversation concerned with unfastening the moorings (times normal), but it is interrupted by a reminder to the $2^{\text {nd }}$ officer (times italic) and a planning conversation with the pilot (courier). 


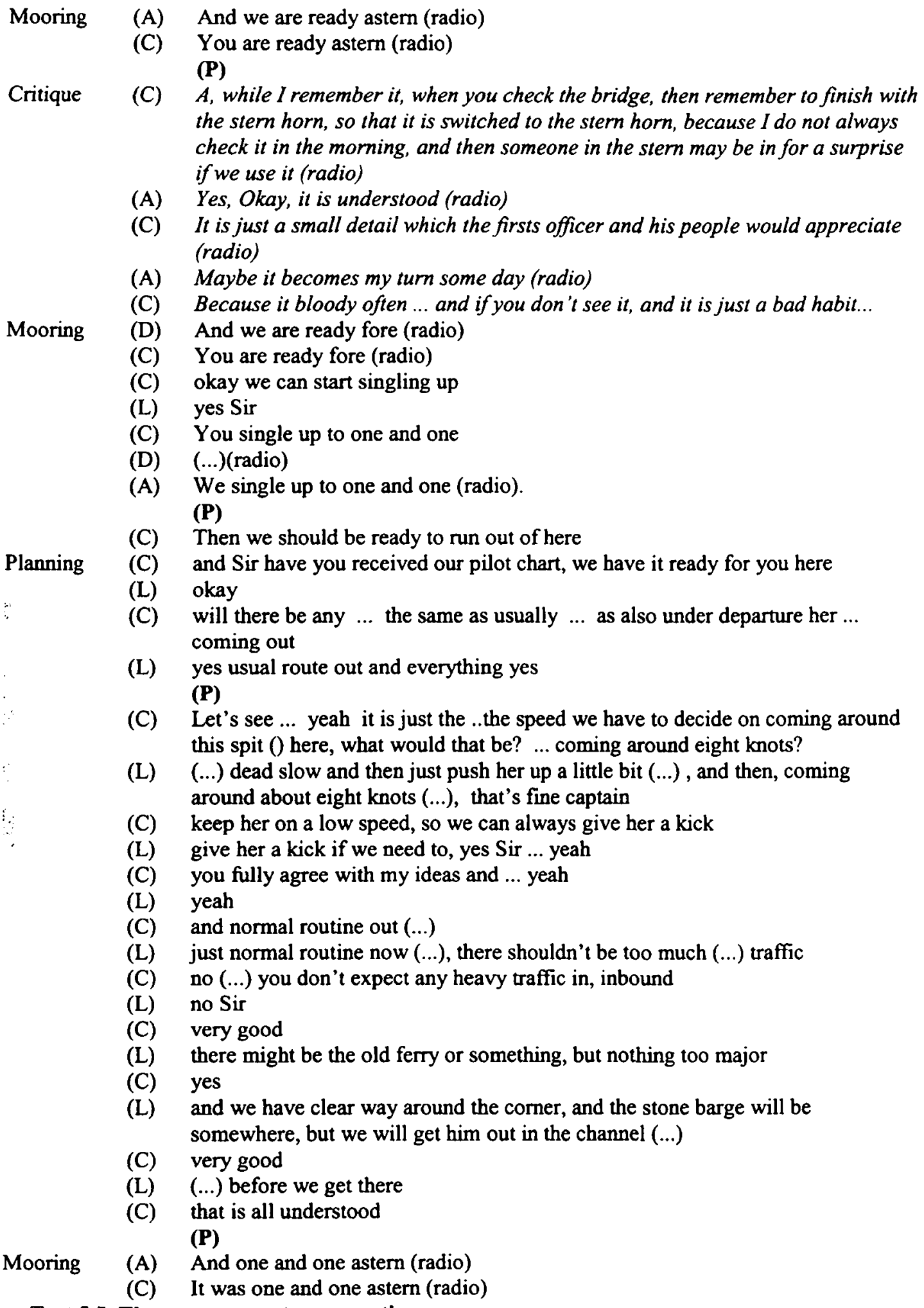

Text 5.5. Three concurrent conversations

\section{Verbalization}

Maritime work must be verbalized and verbalization comes in two forms:

1. It is important that the crew maintains an updated overview of the state of affairs, and for this reason, crew members must verbalize their actions.

2. It is important that errors do not go unnoticed, and it is particularly important that authority does not suppress criticism from subordinates of wrong decisions. 
The two forms differ with respect to control: in the first case, the control action is to maintain a state of knowledge that will deteriorate if communication stops - many pieces of information on the bridge (e.g. the current course and speed, the vessels outside, etc.) loose their validity automatically as the voyage progresses, and must therefore be maintained. In the second case, the problem is not that information becomes invalid, but rather that it is suppressed because of the speaker's fear of his superior. The control action, effected through the ship-owner's guidelines, is thus to suppress this suppression:

In summary the Owners' require superiors as well as subordinate to be assertive and subordinates MUST SPEAK UP when orders in their considered opinion are contrary to rules and regulations or will obviously give results contrary to the superior's intent.

Maersk 1999: Section 2.02: 1-2.

As shown in the italicized words above, the control actions seem useful for describing these important facts too. Fig. 5.1 diagrams the first case, maintaining an up-to-date overview.

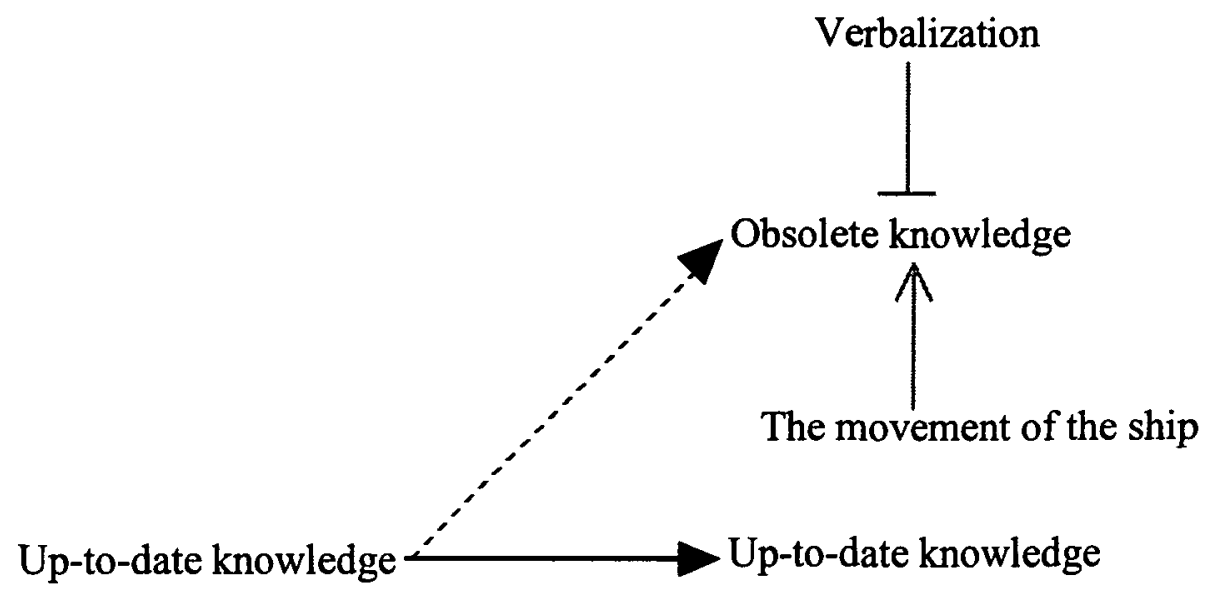

Fig. 5.1. Maintaining overview.

However, such norms can themselves be the victim of obstacles such as forgetfulness or sloppiness, and therefore themselves need to be maintained: maintain in order to maintain. We see this in the next example where the $1^{\text {st }}$ officer notices that the pilot manipulates the autopilot himself. This is not allowed on this ship, since pilots do not verbalize their actions, and thereby violates the rule of verbalization. Therefore the officer notifies the captain of the breach of regulations, and the captain asks the pilot (politely: Nah, it's easier just to lets us now) to ask the crew to change the course.

(D) Now at zero nine zero

(C) Zero nine zero, well he doesn't say anything about it

(D) No, maybe we should ask him to

(C) Yes

\section{(P)}

(D) When you infor... when you changed the course (...) inform (...) just tell me which course you go to please 0

(L) Okay

(D) Thank you very much

(C) Nah it's easier just to lets us now, then we will do it so .... yeah

(L) Okay fine

Text 5.6. Maintaining the rule of verbalization.

In the next example, the helmsman is criticized for not verbalizing when the ship has reached the new course:

(C) And then you sing out, $\mathrm{K}$, when you are on the new course, right, (..) when it is there, then you say one one five, when it is there, then we know, then we know that it is there

(K) I did say that the last time

(C) Well, I just didn't hear it

(K) (...)

(C) Okay, (...), its okay...just keep doing it

Text 5.7. Maintaining the rule of verbalization. 
n the second norm (Fig. 5.2) the crew want to produce relevant criticism if he possess the relevant knowledge. However, the authoritarian tradition endangers this by suppressing producing. A countermeasure is to suppress suppressing producing where the two suppressions are hoped to cancel one another.

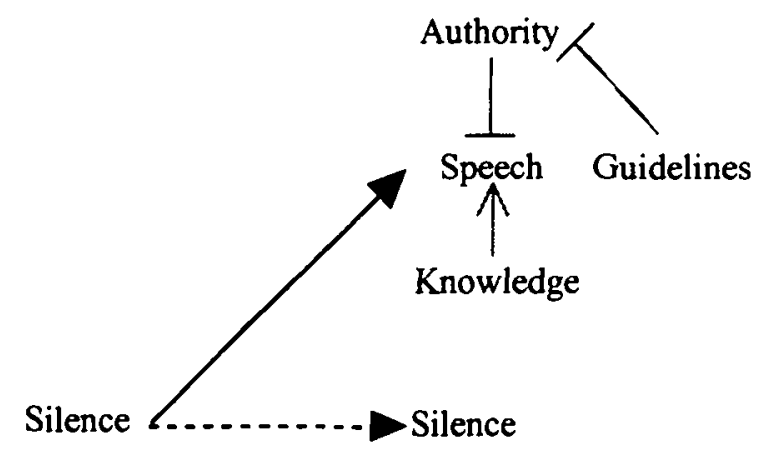

Fig. 5.2. Preventing the suppressing effect of authority.

Although formally, produce $=$ suppress + suppress + produce, the two expressions do not denote the same state of affairs, since in the last case suppression of suppression is not just "cancelled out", but is realized as expensive training courses and written guidelines.

\section{Focus and background}

In any communicative situation, some features are taken for granted, whereas others are the topic of discussion. We shall say that the former constitute the background, the latter the focus. Backgrounded information is either

- not expressed at all,

- referred to via unstressed pronouns or definite nouns that recur identically during the conversation, or

- referred to via certain backgrounding constructions, such as adnominal adjectives and clauses.

Information can be backgrounded for two reasons: either it is irrelevant and can be represented by indefinite pronouns, such as someone or somewhere, or it is already fixed to a specific item, in which case it can be referred to by definite pronouns, such as he, she or it.

We can in fact define a set of communicative functions by means of particular combinations of backgrounded and focused categories of information. The definition can be made very precise by considering a typical sentential schema we assume are used to interpret utterances about physical processes.

\begin{tabular}{|l|l|l|l|l|l|}
\hline Subject & $\begin{array}{l}\text { Tense, Aspect, } \\
\text { Modality }\end{array}$ & Verb & Object & Manner & Time, place \\
\hline someone & does & act on & something & in some way & sometime \\
\hline
\end{tabular}

Schema 5.1. The basic sentential schema.

A sentential schema consists of a set of slots following one another in a more or less fixed sequence. Each slot can be filled with a particular kind of linguistic material, e.g. nouns, verbs or adverbials. A set of items that can fill the same slot is normally called a paradigm.

If we assume that each field can be backgrounded or focused, then there are $2^{6}=64$ different communicative functions. If we furthermore distinguish between three modes of communications, indicative, interrogative and imperative, there are 192 in total. Of these, only a small number are actually found. For example, it does not seem to make sense to have all slots backgrounded.

Below is a list of examples of communicative functions defined by different combinations of background and focus (boldface) paradigms (adapted from Andersen 1997). Focus paradigms are filled with an question pronoun, indicating an empty placeholder that must be filled. Background paradigms come in two varieties: they can also be filled by a indefinite pronoun indicating that the filler of the slot is irrelevant or is postponed to later. The reason for this is the nature of planning. Plans are seldom fully specified but contain undecided elements that are only later filled in. The speaker is consciously vague or currently uninterested in these pieces of information. However, it can also be filled by a definite pronoun indicating that the choice is already made, either by default or previously in the conversation. 


\section{Imperative:}

\begin{tabular}{|l|l|l|l|l|l|}
\hline Who & should begin & acting on & it & in some way & when \\
\hline
\end{tabular}

Schema 5.2. Work distribution: who should begin doing it when? Example: writing out the watch schedule: who should take the watch at which time.

\begin{tabular}{|l|l|l|l|l|l|}
\hline Who & should & act on & it & in some way & sometime \\
\hline
\end{tabular}

Schema 5.3. Resource allocation: who should do it? Example: assigning responsibilities to crew: the engineer is responsible for maintenance of the ship.

\begin{tabular}{|l|l|l|l|l|l|}
\hline $\mathrm{He}$ & should begin & acting on & it & in some way & when \\
\hline
\end{tabular}

Schema 5.4. Scheduling: He should begin doing it when? Example: fixing the time for a job: the $2^{\text {nd }}$ officer and a sailor should be on the deck at 3 o'clock to assist the pilot embarking.

\begin{tabular}{|l|l|l|l|l|l|}
\hline He & should begin & acting on & what & in some way & now \\
\hline
\end{tabular}

Schema 5.5. Object scheduling: He should begin doing it to what now? Example: fixing the exact sequence in which objects are handled: the captain pointing out the individual wires to let go in leaving the quay.

\begin{tabular}{|l|l|l|l|l|l|}
\hline $\mathrm{He}$ & should begin & acting on & what & in some way & sometime \\
\hline
\end{tabular}

Schema 5.6. Task allocation: what should he do to what? Assigning duties to the Philippine maintenance crew: you should paint the fore deck now.

\begin{tabular}{|l|l|l|l|l|l|}
\hline He & should & act on & something & how & sometime \\
\hline
\end{tabular}

Schema 5.7. Instruction: how should he do it? Example: rudder and course orders: the helmsman should turn the wheel which degrees now?

\section{Interrogative:}

\begin{tabular}{|l|l|l|l|l|l|}
\hline He & is & acting on & it & in some way & now \\
\hline
\end{tabular}

Schema 5.8. Supervision: is he doing it?

\begin{tabular}{|l|l|l|l|l|l|}
\hline He & has & acted on & it & in some way & now \\
\hline
\end{tabular}

Schema 5.9. Person Reporting: has he done it? Checking whether the $2^{\text {nd }}$ officer has contacted the pilot.

\begin{tabular}{|l|l|l|l|l|l|}
\hline Someone & has & acted on & it & in some way & now \\
\hline
\end{tabular}

Schema 5.10. Object Reporting: has it been done to it? Checking whether the combination has been taken in after pilot departure.

\begin{tabular}{|l|l|l|l|l|l|}
\hline Someone & has & acted on & it & how & now \\
\hline
\end{tabular}

Schema 5.11. Control check: has it been done to it in the correct way?

In work distribution, the question concerns the actor and the time, while the action and the object are given (who should begin doing it when?). The problem is the who, not the manner and not the what. Therefore the actor and time paradigms should contain many members, while the manner slot is irrelevant. A good maritime example is the watch schedule that determines who will be on the bridge at which time.

Conversely, in instruction the implied question concerns the manner (how should he do it?), and the actor and time slots are irrelevant. An example is the captain's rudder and course commands (Text 5.8), but also his instruction to the helmsman to remember to report back when the new course has been reached.

Scheduling, which is very frequent in maritime operations, differ from work distribution in that the actor too is backgrounded, and the only remaining problem is to fix the time he is to do it.

The difference between person and object reporting is that in the first case, we are interested in the actor's behavior and not in the particular work object, e.g. because we want to know if he is finished and free to do another job, whereas the object's state, and not the person's, is important in the latter case.

The purpose of using background and focus paradigms is to reduce complexity in the sense of Luhmann (Section 1). If the utterance is to specify an action that can be performed, then all paradigms must contain exactly one member that connects to work operations. One cannot make the ship sail "some" course. The course must be " $200^{\circ}, 201^{\circ}$, etc.". So the purpose of the conversation is to fix all focus paradigms to exactly one. But if we can choose between, say 10 items in each slot, there are $10^{6}$ possibilities to choose from, and we will never get around to do anything before the ship is grounded. 
However, if we do neither have to bother with the subject (the helmsman does it), the time (it is now), the action (turn), the object (the wheel), complexity is reduced to the manner slot with a few degrees of freedom (1-30 degrees to each side). Text 5.8 shows an example and Schema 5.12 shows the instruction sentential schema that is used to interpret it.

$\begin{array}{ll}\text { Pilot } & \text { starboard twenty } \\ \text { Helmsm } & \text { What? } \\ \text { Pilot } & \text { starboard twenty } \\ \text { Helmsm } & \text { starboard twenty }\end{array}$

Text 5.8. Rudder commands.

\begin{tabular}{|l|l|l|l|l|l|}
\hline Subject & Modality & Verb & Object & Manner & Time, place \\
\hline Helmsman & should & turn & wheel & $\begin{array}{l}\text { starboard or } \\
\text { port, 1-30 }\end{array}$ & $\begin{array}{l}\text { Now, at the } \\
\text { helm stand }\end{array}$ \\
\hline
\end{tabular}

Schema 5.12. Instruction: how should he do it?

If we look at maritime conversations we discover that typically most of the paradigms are backgrounded by means of the watch schedule, the formal hierarchy, and the voyage plan, so that very few paradigms are focused. The most frequent are the manner (cf. Schema 5.12), the time and the aspect/modality paradigm. Many maritime conversations are about the detailed parameters for handling instruments, the time for doing an action, and whether an action is imminent, executing or ended (aspect).

- Aspect: is ready (about) to do it, begins doing it, is doing it, has done it. Aspect is extremely important in maritime communication. Many tasks are explicitly run through all four phases: for example, when the lines are taken during berthing (Text 5.5), the captain is informed (1) when the deck crew is ready for the operation, and he orders them (2) to start the operation. He may be informed while they are doing it (3), and the crew reports back when they have done it (4).

- Tense and time: past, present, future (will). Tense and time is just as important. The future time of the main operations is regularly discussed.

- Modality: must (is required to), may (is allowed to), must not (is prohibited to), may not (is not required to), possible, and impossible. Course-orders are imperatives, whereas reports are indicatives. Relief from duty is of the "allowed to" variety.

There are three important processes connected with focus: (1) opening the relevant focus paradigms in order to line up the relevant options; (2) closing the focus paradigm in order to reach a decision, and (3) re-focusing (converting background paradigms into focus paradigms) when it is discovered that backgrounded information is erroneous.

Text 3.8 illustrates the latter process. The assumed background action is "passing head-on" and the focused manner slot contains two members, red to red and red to green, from which they have to choose. Sealand Atlantic makes the wrong choice red to red although she ought to know that Sally is turning port and that the action will be "overtaking". The captain begins to re-focus the modality paradigm (how can we) and the correct action (he will overtake us probably) and the Pilot quickly realizes his error (not possible) — although he does not admit to it!

In order to follow the shifting members of focus and background paradigms, we can draw a diagram where the $x$-axis represents time, and the $y$-axis represent the slots to be filled out. We add the various fillers of the slots and record how long they are active. As long as two or more fillers exist for the same slot, the slot is a focus paradigm whose contents have not been decided. When the lifetime of all but one filler has ceased, the slot is unambiguously filled with one option.

Consider again the discussion about the future maneuver in Rotterdam harbor (Text 3.5). What is constant in the sentential schema is the subject which is "Sally Maersk", the place which is "Rotterdam harbor", and the verb which is "turn". These are background slots. The discussion is concerned with the process of entering the harbor, either turning in the Beer Canal or turning inside the basin. There is no question of whether to "turn" or not, the choice concerns the location of the turn, which makes part of the place-adverbial, "inside/outside basin", the focus slot (Fig. 5.3). 


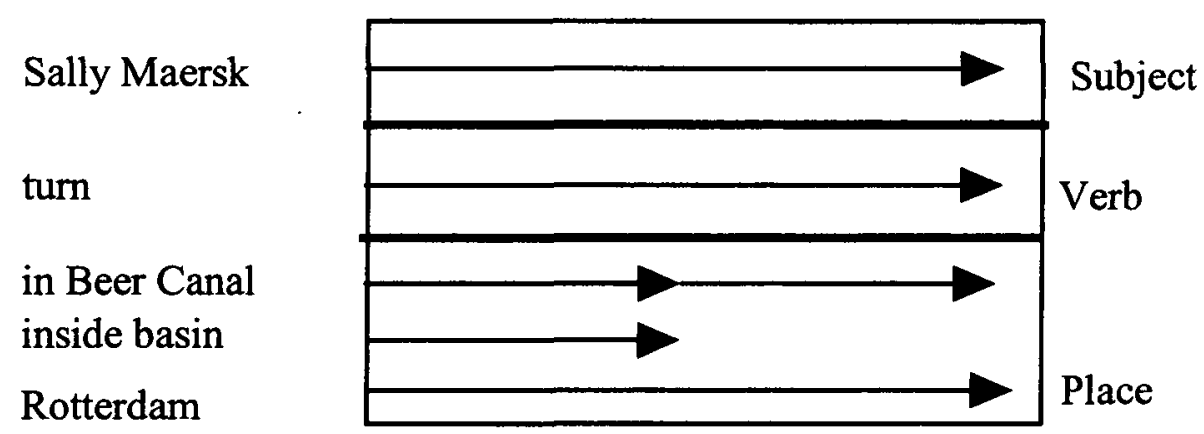

Fig. 5.3 Focus and background. Closing the place slot.

\section{PUTTING IT ALL TOGETHER}

We can now combine the focus-analysis with the action analysis. In both action and communication people try to achieve a desired state or avoid an unwanted one, and in both cases, meaning is a modal concept where the actual is pitted against the possible. However, in communication, the state people try to control is not a physical state, but their shared opinion or consensus. The actions available are not physical actions but communicative moves, and the influence of actions upon the state is not a causal effect, but rather an indirect perturbation of the participants' states of mind. The state that will persist if I do not say anything (the passive projection) is the background paradigms, whereas the change in the focus paradigms is the result of my linguistic action. Under this interpretation, it is easy to find communicative variants of the six basic action types:

(1) Prevent(suppress, avoid): if I hadn't said it, then an undesirable opinion would have developed. Preventing the dangerous effect of authoritarian suppression in Section 5.3.

(2) Maintain: if I hadn't said it, then a desirable opinion would have disappeared. Reminding the $2^{\text {nd }}$ officer of his duty to call the pilot.

(3) Help: a positive opinion would have developed anyway, but $\mathrm{I} /$ something made it happen faster/more efficiently. Captain and pilot cooperating abut the correct maneuvers into the harbor in Text 3.5.

(4) Create (produce): if I hadn't said it, the opinion wouldn't have appeared. Captain commenting on new ships he notices, Text 5.2-5.4.

(5) Destroy(remove): if I hadn't said it, an undesired opinion would have persisted. Captain criticizing bad habits.

(6) Let: it will happen even if I don't do anything. The speaker keeps silent because he agrees to the lines of arguments so far.

As an example of a pure linguistic application, consider the anaphor chain in Text 6.1.

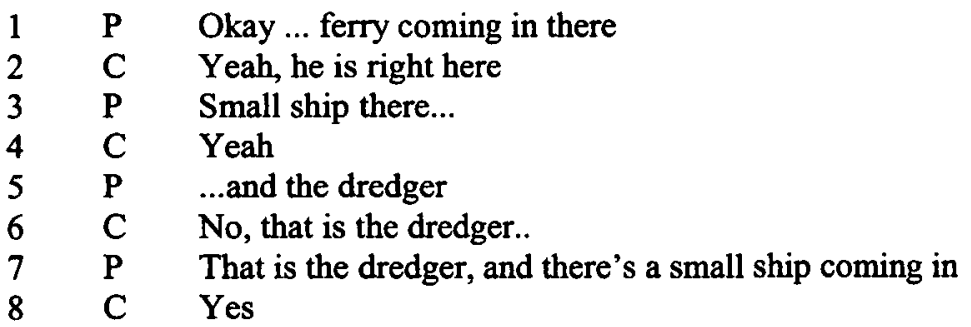

Text. 6.1. Handling references.

As shown in Fig. 6.1, captain (C) and pilot (P) are on their way out of Felixstowe harbor and the captain has just begun to plot traffic ships on the radar. Both pilot and captain keep lookout for traffic via the radar and the window. 


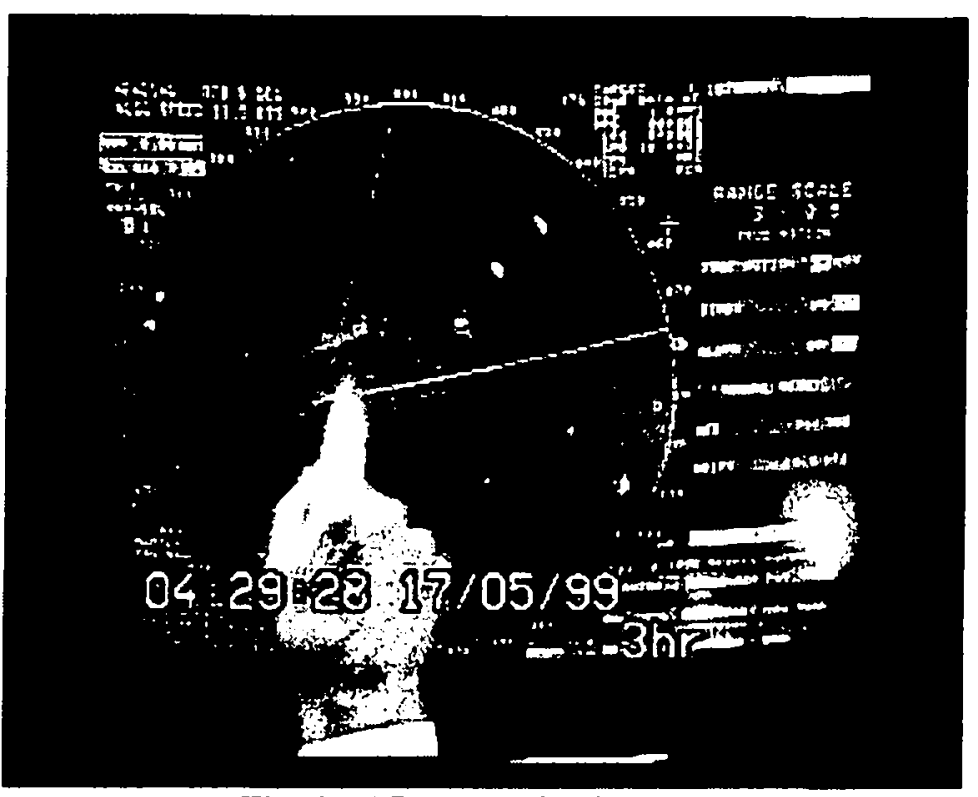

Fig. 6.1. "Ferry coming in there"

In (1) the pilot creates an association between a vessel noun and a reference (possibly by pointing). In (2) the captain maintains this reference by means of the pronoun he, and confirms the observation. In (3) the pilot creates yet another reference, and in (5) the pilot maintains a reference from a previous conversation by means of the definite article (the dredger-unfortunately, I can't find the introduction of the dredger on the tape). However, in (6) the captain suppresses this reference again: the ship pointed to is not the dredger.

The latter two operations are displayed in Fig. 6.2. The interpretation is that first the captain wants to remind himself and the pilot of the previous identification of the dredger that may have been jeopardized by forgetfulness. The pilot thinks that this is a misidentification that should not be allowed to live on, and he therefore suppresses it.

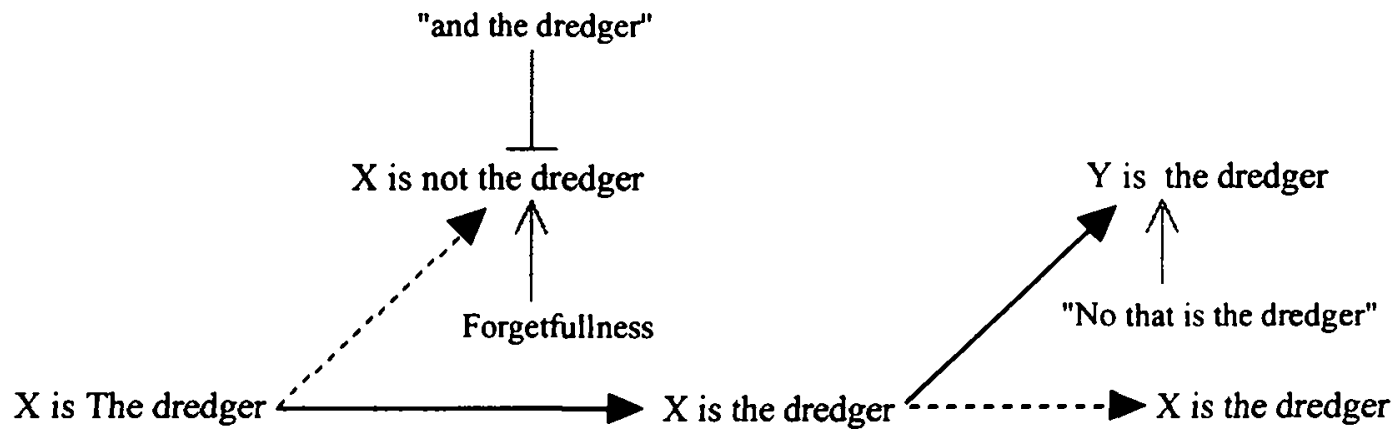

Fig. 6.2. Maintaining and destroying references.

I have chosen a "technical" subject like construction of anaphor chains to substantiate my hypothesis that the action concepts presented may in fact be refined to account for bona fide linguistic processes too.

To summarize, the claims of this paper are: (1) meaning is a modal concept articulating the possible against the actual; (2) both physical actions and communications are controlled by six action types that oppose the passive projection of the current state against active interference; (3) the six action types imply different configurations of helping and opposing forces; (4) the state controlled by communicative actions is represented by means of focus and background paradigms; (5) the framework can describe essential properties of maritime maneuvers, maritime instrumentation and communicative functions.

The paper does not say anything definite about how communication interacts with its social, psychic and physical environment. The focus/background distinction, however, suggests that two processes are important: (6) variety controls variety, i.e. the linguistic choices of the focus paradigms are used to control the choices in the physical and social world; (7) the environment is controlled by reducing its complexity, i.e. the background paradigms represent features of the environment that are taken for granted, and only a small remaining fraction is presented as choices in the focus paradigms. 


\section{REFERENCES}

ANDERSEN. P. BØGH \& M. MAY(2001). Tearing up interfaces. In: Information, organisation and technology. Studies in organisational semiotics. Eds. Kecheng Liu, Rodney J. Clarke, Peter Bøgh Andersen, Ronald K. Stamper. Kluwer: Boston/Dordrecht/London: 299-338.

ANDERSEN. P. BØGH (1997). A theory of computer semiotics. Semiotic approaches to construction and assessment of computer systems. Cambridge: Cambridge University Press, 1990, paperback edition 1997.

ANDERSEN. P. BØGH (1999). Elastic interfaces. Maritime instrumentation as an example. Proceedings of the CSAPC'99, Université de Valenciennes, Valenciennes France, 35-41.

ANDERSEN. P. BØGH (2000). Communication and Work on Maritime Bridges. CHMI-1-00. http://www.cs.auc.dk/ pba/ReportList

CARSTENSEN, P.H. \& M. NIELSEN (1998). SAFECO WP II.1.2: Task analysis of navigator performance. DMI/Risø National Laboratory.

DANISH MARITIME INSTITUTE (1995). Ship handling, Course Manual. Lyngby: Danish Maritime Institute.

LIND, M. (1990). Representing goals and functions of complex systems. Institute of Automatic Control Systems. DTU. 90-D-381.

LIND, M. (1994). Modeling goals and functions of complex industrial plants. Applied Artificial Intelligence 8. 259-283.

LIND, M. (2000). Actions, functions and failures in dynamic environments. CHMI-8-00. http://www.cs.auc.dk/ pba/ReportList

LUHMANN, N. (1990). Essays on Self-Reference. New York: Columbia University Press.

MAERSK (1999). Guidelines for navigators. Maersk Vessels. Technical organisation, level 2 document. NAU610. Version April, 1999

POSNER, R. (1993). Believing, causing, indenting: The basis for a hierarchy of sign concepts in the reconstruction of communication. In: Jorna, van Heusden \& Posner (eds.) Signs, Search and Communication. Berlin: Walter de Gruyter: 215-270.

RYAN, M-L. (1991). Possible Worlds, Artificial Intelligence and Narrative Theory. Bloomington \& Indianapolis: Indiana University Press. 